\title{
Context Model Based on Ontology in Mobile Cloud Computing
}

\author{
Changbok Jang, Euiin Choi* \\ Dept. Of Computer Engineering, Hannam University, Daejeon, Korea \\ chbjang@dblab.hannam.ac.kr, eichoi@hnu.kr
}

\begin{abstract}
Mobile Cloud Computing has become as a new IT paradigm because of the growth of mobile device like smartphone and appearance of Cloud Computing environment. This mobile cloud environment provides various services and IT resources according to users' requests, so an effective management of service and IT resources is required. Hence, this paper designs a context model based on ontology in mobile cloud computing in order to provide distributed IT resources and services to users based on context-awareness information. As the context model proposed in this paper uses context-aware information, it enables to provide more accurate personalized services and manage distributed IT resources.
\end{abstract}

Keywords: Cloud computing, Context-aware, Context model, Ontology, Intelligence Service, Mobile cloud computing

\section{$1 \quad$ Introduction}

The market of mobile recently has been evolving rapidly and cloud computing is spreading into mobile as well. That is why mobile cloud computing is becoming a new issue today. Cloud computing is the computing that provides virtualized IT resources as a service by using Internet technology. In cloud computing, a user lends IT resources (software, storage, server, network) as needed, uses them, get a support of real-time scalability according to service load, and pays as he/she goes. Especially the cloud computing environment distributes IT resources and allocates according to user's request, so there should be a study on technology that manages these resources and deals with effectively[1].

Mobile cloud computing creates a new chance for IT industry because it allows the superiority and economic of cloud computing to meet the mobility and convenience of mobile and draws a synergy effect for both. Also mobile cloud computing refers to an infrastructure that data storage and data processing is done outside mobile device by using cloud computing in the regardless of kinds of mobile devices. Mobile devices used in the mobile environment include personal information and enable to provide the environment that collects a variety of context-aware information. Users' demand on service types suitable for the individual situation has been increasing.

${ }^{*}$ Corresponding Author 
Therefore, context-aware reasoning technique has been studied to provide a suitable service for user by using user' context and personal profile information in mobile environment[2-9]. In this context-aware system, a formal context model has to be provided to offer information needed by application as well as store context and manage. However, there are some technical constraints for this context-aware model to overcome because it itself cannot be applied to mobile platform due to limited device resources, so the study on intelligent mobile service in mobile platform is still insufficient. Recent interest related to mobile cloud is personal smartphone. The study on physical support like connecting smartphone to personal virtual system on cloud and using computing resources unlimitedly is quite active, but the study on how to manage distributed IT resources effectively and provide intelligent mobile service through reasoning based on collected information and role as a medium of collecting context of mobile device is ignored.

Therefore, this paper designs a context model based on ontology in mobile cloud computing and develops in order to the optimized mobile cloud service through recognizing the conditions of user and cloud server and reasoning on the basis of external context achieved from mobile or internal user's personal information and information of resources from cloud server and service use information.

\section{Related Works}

Mobile platform is mainly referred to mobile middleware what lets users operate the optimized contents or service on mobile and it provided a formed interface to UI and service by using RTOS (Realtime OS) and hardware function. There are Windows Mobile, iPhone, Android, Symbian, etc. as these mobile platform.

There are Context-aware information modeling techniques such as Key-value model, Markup scheme model, Graphical model, Object oriented model, and ontology based model which are used in the existing ubiquitous environment and Web environment. Ontology model, a Context-aware model which has been studied mostly recently, enables to express concepts and interactions easily. Recently ontology model has been studied lively related to Semantic Web study based on OWL(Web Ontology Language) and there is a movement to adapt ontology-based model in a variety of context-aware framework. One of the early methods of context modeling using ontology was proposed by Otzturk and Aamodt. Van Heijst divided ontologies into Structure Type and Concept Issues in the study for ontology. Structure Type is classified as Knowledge Modeling Ontology, Information Ontology and Terminological Ontology. Concept Issues is divided as Domain Ontology, Application Ontology, Representation Ontology and Generic Ontology[10]. Guarino classified ontologies according to general level to represent context of different kinds [11]. Top-level Ontologies describe general concepts like space, time, matter, object, event and action. Domain Ontologies and Task Ontologies describe the vocabulary related to a generic domain or a generic task or activity by specializing the terms introduced in the Top-level Ontology. Application Ontologies describe concepts depending both on a particular domain and task, which are often specializations of both the related ontology. These concepts correspond to roles played by domain 
entities while performing a certain activity. Context modeling in context-awareness needs to acquire context initially. Then it is necessary to process modeling to enable acquired context to use. Many projects have used context model with their certain type. Context Toolkit[12] suggested middleware layers that serve to convey to application after acquiring original information and transforming it into any type that application can be understandable. Hydrogen was developed by Hofer[13]. This system is based on hierarchical architecture. This model's representation ability is admirable because it represented model with object-oriented method. But the representation formality is incomplete. Karen's context information model is based on object-oriented method. This modeling concept provides a formal basis for representing and reasoning about some of the properties of context information such as its persistence and other temporal characteristics, its quality and its interdependencies[14]. He attempted to model using both the Entity-Relationship model and the class diagrams of UML. CASS(Context-Awareness Sub-Structure)[15] is a framework for context-aware mobile application designed with middleware approach. By separating into application and context inference, this middleware can be able to infer context without recompiling. CONON(the Context Ontology)[16] is divided as Upper Domain and specific Sub Domain. The context model is structured around a set of abstract entities, each describing a physical or conceptual object including Person, Activity, Computational Entity and Location, as well as a set of abstract sub-classes. This model supports extensibility to add specific concepts in different application domain. It also supports the use of logic reasoning to check the consistency of context information, and to reason over low-level, but it's difficult to represent diverse context with upper context restricted selectively.

However, these context models not sufficient on mobile cloud computing. Therefore, this paper proposes context model in order to manage resources more effectively by using personal context information and do modeling context-aware information in mobile platform and reason.

\section{System Architecture}

In this paper, we suggested context-aware-based intelligence mobile cloud service platform for efficiently managing resource to use context-aware information. 


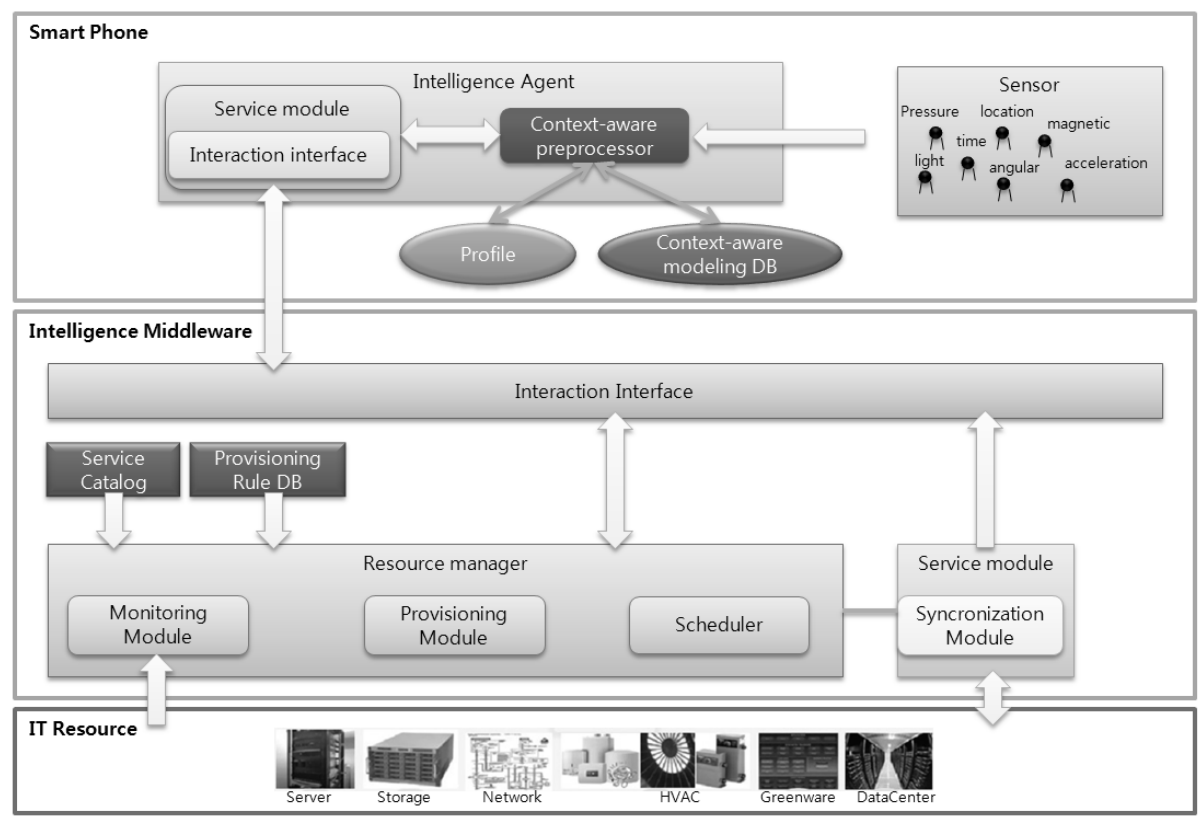

Fig. 1. Suggested platform architecture

As shown figure 1, suggested system consisted of intelligence agent and intelligence middleware. Intelligence agent was responsible for understanding a variety of context-aware information and inferring it. And it consisted of sub-modules such as service module, context-aware preprocessor, personal profile, context-aware information modeling database. Intelligence middleware was responsible for providing services and efficiently managing IT resources by user's request on mobile cloud computing. Context-aware preprocessor on intelligence agent included process for collecting context-aware information and modeling it, inferring context-aware information, and responsible for understanding what user's situation was. Service module was responsible for sending context-aware information to intelligence middleware, providing services that suitable to user. Personal profile was repository which was stored personal information, such as service information by using user, user's ID, password. Context-aware modeling database was stored to information which was modeled by using ontology.

Intelligence middleware consisted of interaction interface for communicating to agent, resource manager, service manager, Service catalog, Provisioning Rule Database. Resource manager responsible for effectively allocating and managing service information was required for processing user's request service, and consisted of monitoring module, provisioning module, and scheduler. Monitoring module crawled information of IT resource utilization.

Provisioning module set up plan for providing best service to analyze contextaware information which was transferred by user and utilization information of IT resource. Scheduler was scheduled to utilization of service and resource by plan which was established to provisioning module. 
Service Catalog was stored service information for which user used, provisioning rule database was stored rule for providing best provisioning process to use contextaware information and utilization of resource. Also, service module was responsible for executing service and using distributed IT resource to providing service to user, and consisted of sub-module, such that synchronization module. Synchronization module responsible for synchronizing resource which user was using on cloud computing.

\section{Classification of Context Model}

In mobile cloud computing, context-aware information which can be used is user's profile, services that user was used, resources for providing services. And we need to provisioning techniques in order to manage resources more effectively on mobile cloud computing, multimodal techniques for supporting convenient user's interface, inferring user's intention more accurately. So, we include entity such as provision, activity. Figure 2 shows each entity and relational property.

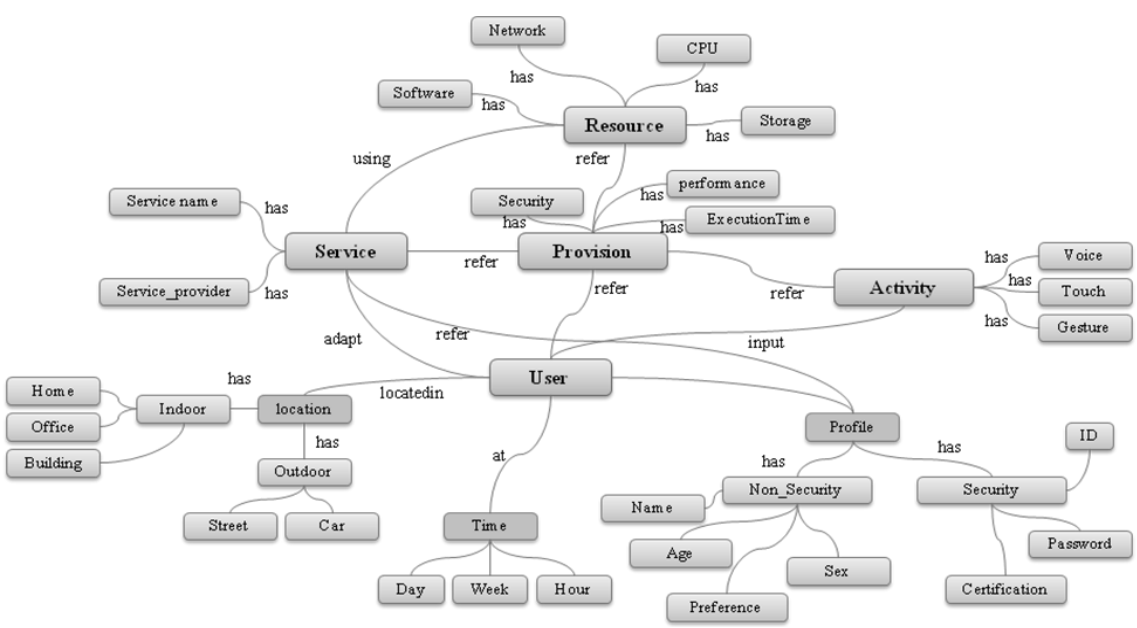

Fig. 2. Context model on mobile cloud computing

In this paper, generic ontology is user, service, resource, provision, activity. And they are connected with each other through relational property(eg. Locatedin between User and location). Individual generic ontology includes domain ontology as a detailed material and immaterial entity(eg, User and location). Consequently, it provides extensibility and formal representation ability by hierarchical ontology classification. 


\section{Conclusion and Future Work}

Context modeling in context-awareness needs to acquire context initially. And then it is necessary to process modeling to enable acquired context to use. In this paper, we have proposed context model to provide users with suitable services and manage resources effectively by using context information in the Mobile Cloud environment. We have also defined context for modeling through diverse context definitions. We have classified ontology and represent hierarchically. The proposed context model by the paper is expected to help have the optimized personalized service and effective IT resources management in the Mobile Cloud environment.

As a further research, we will include additional function for inference. Also we will try to progress in a study that interpret and inference the high level context, and study the resources management technique that manages distributed IT resources effectively by using context information, and the part that examines the performance and tests after embodying the actual platform proposed.

Acknowledgments. This research was financially supported by the Ministry of Education, Science Technology (MEST) and National Research Foundation of Korea(NRF) through the Human Resource Training Project for Regional Innovation

\section{References}

1. AMIT GOYAL and SARA DADIZADEH : A Survey on Cloud Computing, In: University of British Columbia Technical Report for CS 508(2009).

2. Hess, CK., Campbell, RH. : An application of a context-aware file system, In: Pervasive Ubiquitous Computing, Vol. 7, No. 6(2003).

3. Khungar, Sh., Riekki, J. : A Context Based Storage for Ubiquitous Computing Applications, In: Proceedings of the 2nd European Union symposium on Ambient intelligence, pp. 5558(2004).

4. Mayrhofer, R. : An Architecture for Context Prediction, In: Trauner Verlag, Schriften der Johannes-Kepler-Universität Linz, Vol. C45(2005).

5. Byun H.E., K. Cheverst : Exploiting User Models and Context-Awareness to Support Personal Daily Activities, In: Workshop in UM2001 on User Modelling for Context- Aware Applications(2001).

6. Byun, H.E., Cheverst, K. : Utilising context history to support proactive adaptation, In: Journal of Applied Artificial Intelligence, Vol. 18, No 6, pp. 513-532(2004).

7. G. M. Sur, J. Hammer : Management of user profile information in UbiData, In: University of Florida Technical Report TR03-001(2003).

8. Biegel, G., Vahill, V.: A Framework for Developing Mobile, Context-aware Applications. In: IEEE International Conference on Pervasive Computing and Communications (PerCom)(2004)

9. Gu, T., Pung, H.K., Zhang, D.Q.: A Middleware for Building Context-Aware Mobile Services. In: Proceedings of IEEE Vehicular Technology Conference (VTC) (2004).

10. N.Guarino. : Formal Ontology in Information Systems. In : Proceedings of FOIS'98, Trento, Italy, 6-8 June(1998).

11. B.N. Schilit, N. Adams and R. Want. : Context-Aware Computing Applications. In : IEEE Workshop on Mobile Computing Systems and Applications, 8-9 December(1994). 
12. H. Wu, M. Siegel and S. Ablay. : Sensor Fusion for Context Understanding. In : IEEE Instrumentation and Measurement Technology Conference, AK, USA, 21-23 May(2002).

13. T. Hofer, W. Schwingwe, W. Pichler, G. Leonhartsberger and J. Altmann. : Contextawareness on mobile devices - the hydrogen approach. In : Proceedings of the 36th Annual Hawaii International Conference on System Sciences, pp.292-301(2002).

14. S. Fahy and S. Clarke. : A middleware for mobile context-aware applications. In : Workshop on Context Awareness, MobiSys(2004).

15. B. Schilit, M. Theimer. : Disseminating Active Map Information to Mobile Hosts. In : IEEE Network,8(5), pp.22-32(1994)

16. Xiaohang Wang, Daqing Zhang, et al. : Ontology-Based Context Modeling and Reasoning using OWL. In : Workshop on Context Modeling and Reasoning at IEEE International Conference on Pervasive Computing and Communication (PerCom'04), Orlando, Florida, March 14(2004). 